\title{
A cross-sectional study in healthy elderly subjects aimed at development of an algorithm to increase identification of Alzheimer pathology for the purpose of clinical trial participation
}

\author{
Samantha Prins ${ }^{1,2} \mathbb{D}$, Ahnjili Zhuparris ${ }^{1}$, Ellen P. Hart ${ }^{1}$, Robert-Jan Doll ${ }^{1}$ and Geert Jan Groeneveld ${ }^{1,2^{*}}$
}

\begin{abstract}
Background: In the current study, we aimed to develop an algorithm based on biomarkers obtained through nonor minimally invasive procedures to identify healthy elderly subjects who have an increased risk of abnormal cerebrospinal fluid (CSF) amyloid beta42 (A 3 ) levels consistent with the presence of Alzheimer's disease (AD) pathology. The use of the algorithm may help to identify subjects with preclinical AD who are eligible for potential participation in trials with disease modifying compounds being developed for AD. Due to this pre-selection, fewer lumbar punctures will be needed, decreasing overall burden for study subjects and costs.

Methods: Healthy elderly subjects $(n=200$; age 65-70 $(N=100)$ and age $>70(N=100))$ with an MMSE $>24$ were recruited. An automated central nervous system test battery was used for cognitive profiling. CSF AB1-42 concentrations, plasma AB1-40, A 1-42, neurofilament light, and total Tau concentrations were measured. A 1 1-42/140 ratio was calculated for plasma. The neuroinflammation biomarker YKL-40 and APOE $\varepsilon 4$ status were determined in plasma. Different mathematical models were evaluated on their sensitivity, specificity, and positive predictive value. A logistic regression algorithm described the data best. Data were analyzed using a 5 -fold cross validation logistic regression classifier.

Results: Two hundred healthy elderly subjects were enrolled in this study. Data of 154 subjects were used for the per protocol analysis. The average age of the 154 subjects was 72.1 (65-86) years. Twenty-four (27.3\%) were A $\beta$ positive for $A D$ (age 65-83). The results of the logistic regression classifier showed that predictive features for $A \beta$ positivity/negativity in CSF consist of sex, 7 CNS tests, and 1 plasma-based assay. The model achieved a sensitivity of $70.82 \%( \pm 4.35)$ and a specificity of $89.25 \%( \pm 4.35)$ with respect to identifying abnormal CSF in healthy elderly subjects. The receiver operating characteristic curve showed an AUC of $65 \%( \pm 0.10)$.

Conclusion: This algorithm would allow for a 70\% reduction of lumbar punctures needed to identify subjects with abnormal CSF A $\beta$ levels consistent with AD. The use of this algorithm can be expected to lower overall subject burden and costs of identifying subjects with preclinical AD and therefore of total study costs.
\end{abstract}

\footnotetext{
* Correspondence: GGroeneveld@chdr.nl

${ }^{1}$ Centre for Human drug Research, Leiden, the Netherlands

${ }^{2}$ Leiden University Medical Center, Leiden, the Netherlands
}

C C The Author(s). 2021 Open Access This article is licensed under a Creative Commons Attribution 4.0 International License, which permits use, sharing, adaptation, distribution and reproduction in any medium or format, as long as you give appropriate credit to the original author(s) and the source, provide a link to the Creative Commons licence, and indicate if changes were made. The images or other third party material in this article are included in the article's Creative Commons licence, unless indicated otherwise in a credit line to the material. If material is not included in the article's Creative Commons licence and your intended use is not permitted by statutory regulation or exceeds the permitted use, you will need to obtain permission directly from the copyright holder. To view a copy of this licence, visit http://creativecommons.org/licenses/by/4.0/ The Creative Commons Public Domain Dedication waiver (http://creativecommons.org/publicdomain/zero/1.0/) applies to the data made available in this article, unless otherwise stated in a credit line to the data. 
Trial registration: ISRCTN.org identifier: ISRCTN79036545 (retrospectively registered).

Keywords: Alzheimer, Preclinical AD, Clinical trial, Algorithm, CSF A $\beta$

\section{Background}

As new disease-modifying therapies for Alzheimer's disease (AD) enter clinical trials, identifying the disease at a clinical stage where the pathological injury is not too severe to allow functionally meaningful recovery, or at least stabilization, is a major issue of current research [1]. Classification criteria aim at defining early clinical, biochemical, and metabolic markers of $\mathrm{AD}$ before the clinical criteria of dementia are fulfilled [2]. Identification of the pre-dementia phase of $\mathrm{AD}$ is crucial to allow progress of new treatments designed to intervene in the disease process at the earliest possible stage.

The current leading hypothesis regarding the pathophysiology of $\mathrm{AD}$ is centered on the misfolding and aggregation of toxic amyloid beta $(A \beta)$ species such as A $\beta 1-42$, and drug research has therefore so far focused most on this therapeutic target. Emerging data in otherwise healthy elderly individuals suggest that biomarker evidence of $\mathrm{A} \beta$ accumulation and neurofibrillary tangles are associated with functional and structural brain alterations, consistent with the patterns of abnormality seen in patients with mild cognitive impairment (MCI) and even $\mathrm{AD}$, prior to the clinical expression of symptoms [3]. A phase one study in 2016 showed promising results of the anti-A $\beta$ antibody aducanumab in patients with prodromal and mild $\mathrm{AD}$ by decreasing $\mathrm{A} \beta$ plaques in the brain [4]. Following this phase one study, the compound was further studied in two identically designed phase 3 trials. In March 2019, the trial was halted due to ineffectiveness. Further analyses showed that in one of the two phase 3 trials the patient group that received the highest dose of the active compound showed slower cognitive decline than the placebo group [5]. Based on these results, the FDA recently approved aducanumab for the treatment of AD in the USA under the "accelerated approval pathway" which provides patients access to drugs when there is an expectation of clinical benefit despite some uncertainty about the clinical benefit of the drug [6]. A $\beta$ immunotherapy could prevent (progression to) $\mathrm{AD}$ in healthy elderly who show evidence of amyloid pathology and could prevent (further) aggregation of neurotoxic forms of $A \beta$ and would thereby prevent downstream effects as synaptic dysfunction, neuronal damage, and cognitive impairment [7]. However, many phase 3 anti-amyloid trials have failed to demonstrate effects on progression of cognitive decline in patients with (mild to moderate) $\mathrm{AD}$, despite clear $\mathrm{A} \beta$ lowering effects in cerebrospinal fluid (CSF) or PET [8-12].
Based on extensive longitudinal biomarker studies $[13,14]$, a specific pattern of deterioration of AD specific biomarkers has been proposed, which reflects the underlying progressive neuropathology of the disease. In this model, described by Jack et al., [14] concentrations of $\mathrm{A} \beta$ in CSF start decreasing decades before clinical symptoms appear. Changes in total and phosphorylated tau (t-Tau, p-Tau) concentrations in CSF have been shown to occur up to 15 years prior to the clinical onset of AD $[15,16]$. Studies with $A \beta$ lowering compounds are increasingly performed in cognitively healthy subjects with a CSF profile consistent with $\mathrm{AD}$ or "preclinical $\mathrm{AD}$," due to this early decrease of $A \beta$ in CSF and the hypothesis that cognitive deterioration can still be prevented at this stage $[17,18]$. Over the age of 65 , approximately $20 \%$ of cognitively healthy subjects can be expected to have a CSF profile with lowered $A \beta$ levels consistent with $\mathrm{AD}$ as this is shown to be an age-related process [19]. This means that to identify a single healthy elderly subject with CSF values consistent with $A D$, four subjects will have to undergo a lumbar puncture unnecessarily. This leads to unnecessary overall burden for study subjects and to higher study costs.

In the clinical setting, the diagnosis of (probable) $\mathrm{AD}$ is made based on clinical symptoms (e.g., self-reported memory loss, partner reports, difficulties in daily functioning), combined with neuropsychological testing, and confirmed by evidence of amyloid pathology in CSF (abnormal $A \beta$ and/or Tau levels) or on amyloid PET scans, when available. The collection of CSF is, however, an invasive technique, which is burdensome in itself but also carries a risk of adverse effects (e.g., post-puncture headache) while PET scans are time consuming, not available for all patients, and expensive [19, 20].

As a result of the aforementioned, many studies have attempted to identify blood assays which can reliably measure AD-related biomarkers [21, 22]. Some seem to be successful in making a distinction between blood $A \beta$ levels in subjects with (subjective) cognitive impairment, $\mathrm{MCI}$, or $\mathrm{AD}[23,24]$. Also, the biomarkers $\mathrm{t}$-Tau and neurofilament light (NfL) have been able to make this distinction [25, 26]. Limitations of the current blood-based biomarkers are that outcomes are not consistent between studies and the methods used are highly diverse [27].

In the current study, we aimed to develop an algorithm based on minimally invasive biomarkers (plasma 
and cognitive tests), to be used for pre-selection of subjects with an increased risk of lowered, abnormal, CSF $A \beta$ levels ("A $\beta$ positive subjects") consistent with the presence of $\mathrm{AD}$ pathology. This algorithm can be used to preselect cognitively healthy $A \beta$ positive people for drug studies in preclinical AD, thereby resulting in fewer subjects needing to undergo a lumbar puncture.

\section{Methods}

This was a single-center, cross-sectional, observational, correlational study. All study participants visited the research unit twice, once for a medical screening and once for the study measurements.

The study was approved by the ethics committee of the Leiden University Medical Center (LUMC), the Netherlands. The study was conducted according to the Dutch act on Medical Research Involving Human Subjects (WMO) and in compliance with Good Clinical Practice (ICH-GCP) and the Declaration of Helsinki. The trial was retrospectively registered in the international trial register with ID number: ISRC TN79036545.

\section{Participants}

We aimed to enroll 200 healthy male and female participants, with an age of 65 years and older. Of these 200 subjects, at least 100 participants were to be above the age of 70. All the subjects visited Centre for Human Drug Research (CHDR) between October 2017 and November 2018 where all study assessments took place. CHDR is a clinical pharmacology research facility where early phase clinical drug studies and methodology and biomarker research are performed. For this study, a population of healthy elderly subjects aged 65 years and over was chosen as the prevalence of neurodegenerative disorders with an important cognitive component such as AD increases significantly from this age onwards [19]. Main exclusion criteria were a diagnosis of a cognitive disorder (including but not limited to $\mathrm{MCI}, \mathrm{AD}$, Lewy body dementia, frontotemporal dementia), history of psychiatric disease in the past 3 years, Mini Mental State Examination $(\mathrm{MMSE}) \leq 24$, Geriatric Depression Scale $(G D S) \geq 6$, presence of drug or alcohol abuse $(<2$ standard drinks per day for female and $<3$ standard drinks per day for male), and any medication which influences the central nervous system or is contraindicative for the performance of a lumbar puncture.

All subjects underwent medical screening, including medical history, physical examination, vital signs measurements in supine and standing position, routine hematology, urinalysis, and urine drug screen.

All subjects visited the clinical research unit once for the study day and underwent blood sampling at predefined time points $(0,2$, and 4 hour[s]). A single lumbar puncture was performed for the collection of CSF (at $4 \mathrm{~h}$ ). Furthermore, a CNS test battery was performed to collect data on different CNS domains.

\section{Blood sampling}

Approximately $10 \mathrm{~mL}$ blood was collected via an i.v. catheter placed in an antecubital vein in the arm in appropriate K2EDTA tubes at the predefined time points mentioned above. Immediately following collection of the required blood volume, the tubes were slowly tilted backwards and forwards (no shaking) to bring the anticoagulant into solution. The blood plasma samples for bioanalysis were centrifuged within $1 \mathrm{~h}$, at $2000 \mathrm{~g}$ for 10 min at $4{ }^{\circ} \mathrm{C}$. Prior to centrifugation, plasma samples were kept at room temperature. Immediately after centrifugation, supernatant was divided into $0.5 \mathrm{ml}$ aliquots in Sarstedt polypropylene $0.5 \mathrm{~mL}$ tubes and stored at $80^{\circ} \mathrm{C}$.

\section{Lumbar puncture}

A CSF sample of $4 \mathrm{~mL}$ was collected in a $10 \mathrm{~mL}$ polypropylene tube. CSF was centrifuged within $1 \mathrm{~h}$, at $2000 \mathrm{~g}$ for $10 \mathrm{~min}$ at $4{ }^{\circ} \mathrm{C}$. Prior to centrifugation, CSF samples were kept at room temperature. Immediately after centrifugation, samples were divided into $0.5 \mathrm{ml}$ aliquots in Sarstedt polypropylene $0.5 \mathrm{~mL}$ tubes and stored at $80^{\circ} \mathrm{C}$. Lumbar punctures were performed by a trained, physician with a $25 \mathrm{G}$ atraumatic lumbar puncture needle (Braun, 25G) under supervision of an experienced neurologist. The needle was placed at the L3-L4 or L4-L5 interspace with the subject in supine or sitting position. If a subject suffered from post-dural headaches, the subject was treated according to our standard operating procedures.

\section{Amyloid status}

Amyloid beta1-42 was measured in the CSF using the fully automated Elecsys platform as this is widely used for diagnostics [28]. Lowered $A \beta$ levels classified as amyloid abnormal and consistent with the presence of Alzheimer pathology were dichotomized by creating a group of "A $\beta$ positive subjects" $(A \beta<1000 \mathrm{pg} / \mathrm{ml})$ and "A $\beta$ negative subjects" $(A \beta \geq 1000 \mathrm{pg} / \mathrm{ml})$.

\section{Plasma analysis}

Several plasma analyses were performed in plasma samples that were taken within $1 \mathrm{~h}$ from the CSF sample. Plasma biomarkers have been selected based on promising previous research of the use of plasma biomarkers to predict AD pathology. Although analytical methods vary, previous research has been able to measure $A \beta$, t-tau and NfL in plasma and have therefore been included to this study and the algorithm [23-26]. Plasma concentration of $A \beta 1-40, A \beta 1-42$, $t-T a u$, and NfL were measured 
using the fully automated, highly sensitive single molecule array Simoa technology [29]. The A $\beta$ scores have been used as single variables as well as in a ratio score A $\beta$ 1-42/A $\beta$ 1-40.

Chitinase 3-like 1 (CHI3L1), or more commonly called YKL-40, is a glycoprotein which is mainly expressed in astrocytes. Insoluble $A \beta$ aggregates in the brain can induce the activation of microglia, resulting in the synthesis of proinflammatory mediators, which further can stimulate astrocytic expression of YKL-40 [30]. Higher concentrations of YKL-40 were found in patients with prodromal $\mathrm{AD}, \mathrm{MCI}$, and full-blown $\mathrm{AD}[31,32]$ when measured in CSF. Measuring YKL-40 in plasma can lead to a less invasive method of measuring inflammation related to $\mathrm{AD}$ in healthy subjects. YKL-40 was measured in plasma samples using the CHI3L1 Human ELISA Kit (Thermo Fisher).

\section{Apolipoprotein E genotyping}

Apolipoprotein E (APOE) genotyping was performed after isolating DNA from EDTA blood. DNA was isolated using QIAamp DNA Blood MINI kit after which a polymerase chain reaction (PCR) technique was applied on the clean DNA. A sequential analysis (according to the Sanger method) than determined the APOE genotype. One or 2 APOE $\varepsilon 4$ alleles classified subjects as APOE $\varepsilon 4$ carriers, when no APOE $\varepsilon 4$ alleles were present a subject was classified as noncarrier.

\section{Cognitive assessments and questionnaires}

The NeuroCart is a battery of CNS tests used to assess a wide range of CNS domains [33]. All measurements were performed in a quiet room with ambient illumination. Per session, there was only one participant in the room. The following tests were performed using the NeuroCart: the Adaptive tracking test to measure attention and eye-hand coordination [34], the Face encoding and Recognition task (FACE) to measure visual memory [35], the Visual Verbal Learning Test (VVLT, 30 words) to measure the whole scope of learning behavior (i.e., acquisition, consolidation, storage and retrieval) [36], the Milner Maze test (MMT) to evaluate visuospatial working memory [37], the N-Back test to evaluate working memory [38], the Sustained Attention to Response Task (SART) as a vigilance task [39], and finger tapping for motor fluency [40], and saccadic and smooth eye movement [41] were also measured.

21-Leads electro encephalography (EEG) [42] recordings were made for all subjects to monitor (abnormal) brain activity. An 8-min resting EEG was performed while the subjects alternated $4 \mathrm{~min}$ with their eyes closed and $4 \mathrm{~min}$ with their eyes opened while resting in a chair. Subjects face a featureless wall and are instructed not to stare, not to move their head and eyes, and to suppress eye blinks. The Refa-40 (TMSi B.V., the Netherlands) recording system and 32-lead cap (TMSi B.V.) have been used. The five standard EEG band have been analyzed: delta $(1.5<6.0)$, theta $(6.0<8.5)$, alpha $(8.5<12.5)$, beta $(12.5<30$.$) , and gamma (30.0<40.0)$.

The clinical dementia rating scale (CDR) [43] was assessed via a semi-structured interview with the participating subject only, to rate impairment in six different cognitive categories (memory, orientation, judgment and problem solving, community affairs, home and hobbies, and personal care). To rate impairment in more complex daily activities, the Instrumental Activities of Daily Living Scale (IADL) [44] was assessed. Both questionnaires were administered by trained neuropsychologists.

\section{Sample size justification}

In this study, we selected elderly at the age of 65 years old and higher of which at least a hundred above the age of 70. According to Jansen et al. [19], we expected at least $19 \%$ amyloid pathology in a $65+$ population and $23 \%$ amyloid pathology among cognitively healthy $70+$ elderly subjects. We expected more responsiveness for study participation from elderly between the age of 65 and 70, based on our experience with previous studies with participants in this age range. Participants in this age range have participated in studies at CHDR before and are therefore registered in our local database and have received emails about this study. A higher number of participants within the age range $65-70$ are present in the database compared to older elderly. Therefore, we aimed to enroll at least 100 subjects of $>70$ years old in this study as prevalence of amyloid pathology is expected to be higher in this age group. This would result in an estimated $23 \mathrm{~A} \beta$ positive subjects versus approximately $77 \mathrm{~A} \beta$ negative subjects in the $>70$ years old age group. Along with approximately $19 \mathrm{~A} \beta$ positive subjects versus $81 \mathrm{~A} \beta$ negative subjects in the age group $65-70$, we expected to identify at least $42 \mathrm{~A} \beta$ positive healthy elderly subjects among the total group of 200. Based on previous comparable studies, these numbers were considered appropriate for a correlational study aimed at defining an algorithm $[45,46]$.

\section{Statistical analysis}

Statistical analyses were performed using Python (version 3.7.3) and the sklearn package (version 0.21.3). To build a classification model that could differentiate between $A \beta$ positive subjects and $A \beta$ negative subjects, all parameters such as plasma data, genetic information, cognitive assessments, level of education, age, and gender were included as features.

When a classifier contains more features than can be justified by the observed data, there is a risk of the model overfitting. Overfitting occurs when a classifier corresponds too 
closely to a particular subpopulation and cannot be generalized to the wider population. Two methods were used to reduce the feature space, variance inflation factor (VIF) and penalized regression. VIF identified the pairs of highly correlated features and subsequently removes one of the features from the classifier. Penalized regression was applied to the logistic regression classifier to shrink the coefficients of features that were less predictive of the outcome.

For this study, we reviewed the performance of four classifiers-ridge-penalized logistic regression, random forest classifier, support vector machine classifier, and k-nearest neighbors classifier-on four datasets-a dataset with all features, only the VIF-selected features, all features except the EEG features, and all features except the genotyping feature. To ensure that the models were not under- or overfitting, we performed 5-fold stratified cross-validation. This data partitioning approach ensures that we built a more generalized model that can perform well when presented with unseen data. The 5-fold stratified cross-validation randomly samples the data into 5 folds of approximately equal proportions. In this case, there were 30 or 31 subjects per fold. Each fold contained the same ratio of $A \beta$ positive and $A \beta$ negative subjects. The model was trained on 4 folds of data and validated on the 5th fold. The cross-validation process was repeated 5 times, with each of the subsamples used exactly once as the validation data. The validation results were averaged over each iteration to estimate the model's predictive performance. We selected the optimal classifier by selecting the classifier with the highest sensitivity and specificity. If the sensitivity and specificity scores were identical between classifier, we then choose the classifier with the highest F1 score.

\section{Results}

\section{Demographic and clinical characteristics}

Two hundred healthy elderly subjects were enrolled in this study of which 189 were included in the CSF and plasma analyses due to CSF availability. The 11 missing CSF samples were due to absent CSF flow during lumbar puncture. The 189 CSF samples were analyzed on A $\beta 42$ using the Elecsys method and 55 healthy elderly had CSF $A \beta 42$ levels consistent with $A D(A \beta<1000 \mathrm{pg} / \mathrm{ml})$. Of the 189 subjects with CSF availability, 154 subjects were included in the per protocol analyses. Plasma analyses were missing for 27 subjects due to analytic errors. NeuroCart data was incomplete for 8 subjects. Fortynine subject were female $(68.2 \%$ were male and $31.8 \%$ female). Their mean age was 72.1 years (range 65-86), with a median MMSE score of 29 (range 25-30), and GDS score of 0 (median, range 0-5). Self-reported memory performance and daily functioning were assessed using CDR and IADL scores with averaged scores of 0 in all subjects. Of the 154 elderly, 42 (27.3\%) were $A \beta$ positive for $\mathrm{AD}$ (average age 73.7 [65-83]; see Table 1).

\section{Data analysis}

For each dataset and classifier, we calculated the sensitivity, specificity, precision and F1 score. The VIFselected features dataset and logistic regression classifier achieved a sensitivity and specificity of $70.8 \%$ and $89.2 \%$. The receiver operating characteristic (ROC) curve showed an AUC of $65 \%$ ( \pm 0.10); see Fig. 1. However, the mean performance for all four classifiers (for the VIF-selected features dataset) was $68.0 \%$ sensitivity and $76.4 \%$ specificity. The lowest sensitivity and specificity for the worse performing model (the random forest) was $63.6 \%$ and $70.6 \%$ respectively. While we found similar performance when applying different classifiers, the logistic regression showed the highest sensitivity and specificity for the classification task.

The best performing classifier, logistic regression, included 32 of the 90 parameters measured in this study. The results of the logistic regression algorithm analyses

Table 1 Demographics, clinical characteristics, and biomarker information of the study population

\begin{tabular}{llll}
\hline Characteristics & $\begin{array}{l}\text { Total group, } \\
\boldsymbol{n}=\mathbf{1 5 4}\end{array}$ & Amyloid status CSF & A $\boldsymbol{\beta}$ negative, $\boldsymbol{n}=\mathbf{1 1 2}(\mathbf{7 2 . 7 \% )}$ \\
\hline Age, years & $72.1[65 ; 86]$ & $73.7[65 ; 85]$ & $71.4[65 ; 86]$ \\
Female gender & $49(31.8 \%)$ & $13(30.6 \%)$ & $36(32.1 \%)$ \\
MMSE & $29(25-30)$ & $29(25-30)$ & $29(25-30)$ \\
GDS & $0(0-5)$ & $1(0-5)$ & $0(0-5)$ \\
CDR & $0.0(0-0.5)$ & $0.0(0)$ & $0.0(0-0.5)$ \\
IADL & $0.0(0)$ & $0.0(0)$ & $0.0(0)$ \\
Education ${ }^{a}$ & $6(1-7)$ & $6(1-7)$ & $6(1-7)$ \\
Apoe e4/e4 $(n=150)$ & $5(3.3 \%)$ & $5(100 \%)$ & $0(0 \%)$ \\
Apoe at least one e4 allele $(n=150)$ & $39(26 \%)$ & $18(42.9 \%)$ & $21(18.8 \%)$ \\
\hline
\end{tabular}

Continuous data are presented as mean [min; max] and dichotomous data as n (\%). MMSE, Mini Mental State Examination; GDS, Geriatric Depression Scale; CDR, Clinical Dementia rating Scale; IADL, Instrumental Activity of Daily Living scale; Apoe e4, apolipoprotein E 4.

aLevel of education defined as (1) lower than primary school, (2) primary school, (3) less than lower professional education, (4) lower professional education, (5) mid-level professional education, (6) high school/college, and (7) university 


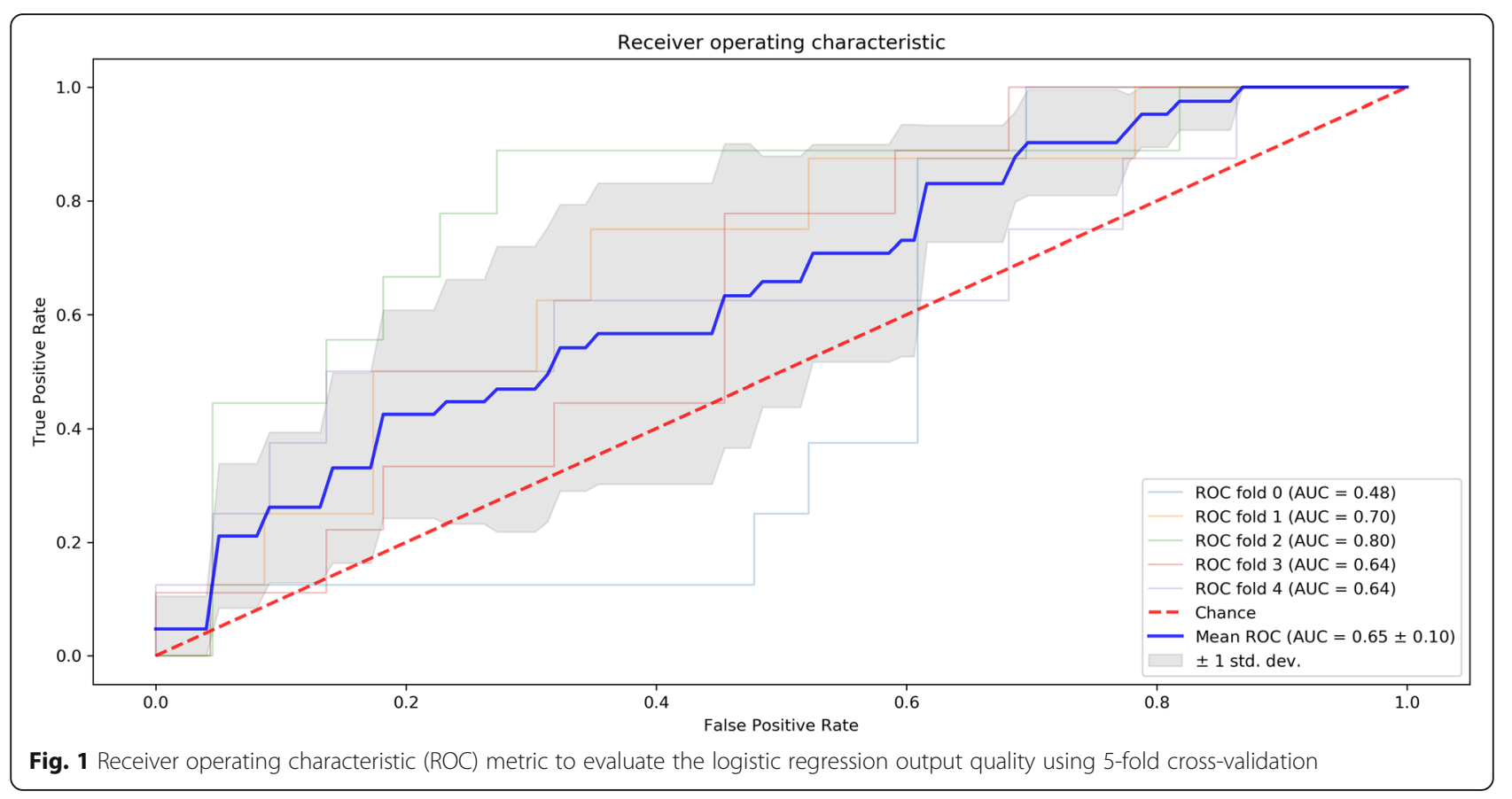

Table 2 NeuroCart activities and parameters included in the algorithm

\begin{tabular}{|c|c|c|}
\hline Activity & Cognitive domain & Parameter \\
\hline Visual Verbal Learning Test (WLT, 30 words) & Memory & $\begin{array}{l}\text { - Delayed word recall number correct } \\
\text { - Immediate word recall number doubles, 3e trial } \\
\text { - Immediate word recall number incorrect 1st trial } \\
\text { - Delayed word recall number doubles } \\
\text { - Immediate word recall number doubles, 2e trial } \\
\text { - Immediate word recall number doubles, 1st trial } \\
\text { - Immediate word recall number incorrect 3e trial } \\
\text { - Delayed word recognition number incorrect } \\
\text { - Immediate word recall number incorrect 2e trial }\end{array}$ \\
\hline Electroencephalography (EEG) & Electrical brain activity & $\begin{array}{l}\text { - Delta-power Fz-Cz (eyes open) } \\
\text { - Theta-power Fz-Cz (eyes closed) } \\
\text { - Beta-power Fz-Cz (eyes open) } \\
\text { - Gamma-power Pz-O2 (eyes open) } \\
\text { - Delta-power Pz-O2 (eyes open) } \\
\text { - Gamma-power Pz-O1 (eyes closed) } \\
\text { - Alpha-power Fz-Cz (eyes open) } \\
\text { - Theta-power Pz-O1 (eyes open) } \\
\text { - Gamma-power Fz-Cz (eyes open) } \\
\text { - Alpha-power Pz-O1 (eyes closed) }\end{array}$ \\
\hline Finger Tapping & Motor activation and fluency & - Standard deviation of the mean (dominant hand) \\
\hline Sustained Attention to Response Task (SART) & Vigilance & $\begin{array}{l}\text { - Total omission errors } \\
\text { - Post error slowing }\end{array}$ \\
\hline N-Back & Working memory & - Number correct-number incorrect/total for one back \\
\hline Milner Maze test (MMT) & Spatial working memory & $\begin{array}{l}\text { - Reversed total illegal moves } \\
\text { - Immediate total repeat errors } \\
\text { - Immediate total illegal moves } \\
\text { - Delayed total illegal moves } \\
\text { - Reversed total repeat errors } \\
\text { - Delayed total repeat errors }\end{array}$ \\
\hline Face encoding and recognition task (Face) & Episodic memory & - Number incorrect \\
\hline
\end{tabular}


conclude that the best prediction of $\mathrm{A} \beta$ positivity/negativity in CSF in an elderly subject is made by combining the 32 parameters measured with the NeuroCart (Table 2). The algorithm included the following 7 CNS tests and 1 plasma analysis: MMT, VVLT, finger tapping, N-Back, SART, Face, EEG, and the plasma biomarker YKL-40. Sex was also included. We can use the logistic regression equation to calculate the probability (between 0 and 1 ) of a new subject being classified as amyloid positive or negative. If the subject is given a probability greater than 0.5 , they will be classified as amyloid positive.

As EEG- and genotyping-based assessments are time and resource consuming tasks, we built two additional classification models excluding these features. By excluding the EEG features, the highest sensitivity and specificity achieved was $70.6 \%$ and $73.5 \%$, respectively, using ridge-penalized logistic regression classifier. Hence, the exclusion of the EEG features had little to no effect on the sensitivity of the classifier but lead to a 15 percentage point drop in specificity compared to the best performing logistic regression model. When omitting the genotyping features (the APOE E4 status), the best performing model was the k-nearest neighbor. This model achieved a sensitivity and specificity of $70.4 \%$ and $72.3 \%$ respectively. Like the classifier with no EEG features, the exclusion of the genotyping data had little to no effect on the classifier's sensitivity, while the specificity did drop by 16 percentage points compared to the best performing logistic regression model.

When aiming for 50 healthy elderly with $A \beta$ CSF levels consistent with $\mathrm{AD}, 220$ elderly must undergo the (non-invasive) tests included in the algorithm. Of these 220 subjects, the algorithm will predict 66 elderly with $A \beta$ positive levels in CSF, 50 of which will be true positives (A $\beta$ CSF levels consistent with $A D$ ), the remaining 16 will be false positive ( $A \beta$ negative). However, $21 \mathrm{~A} \beta$ positive subjects will be mislabeled as $A \beta$ negative (see Table 3 ). This algorithm would allow for a $70 \%$ reduction of lumbar punctures needed to identify subjects with abnormal CSF A $\beta$ levels consistent with $\mathrm{AD}$, meaning 66 lumbar punctures instead of 220 (see Fig. 2).

Table 3 Sensitivity/specificity table of the logistic regression algorithm

\begin{tabular}{llll}
\hline & Predicted $\mathbf{A} \boldsymbol{\beta}+$ & Predicted $\mathbf{A} \boldsymbol{\beta}$ - & Total \\
\hline Actual $A \beta+$ & 50 & 21 & 71 \\
Actual $A \beta-$ & 16 & 133 & 149 \\
Total & 66 & 154 & 220 \\
\hline
\end{tabular}

Sensitivity and specificity table calculated with a sensitivity of $70.82 \%$ and specificity of $89.25 \%$. When aiming for 50 positively predicted $A \beta$ positive subjects, 66 will be predicted as such. Therefore, 16 subjects will falsely be predicted as being $A \beta$ positive and 21 will falsely be predicted as being $A \beta$ negative

\section{Discussion}

This study aimed to develop an algorithm based on lessinvasive (plasma) biomarkers for $\mathrm{AD}$ pathology, to be used for pre-selection of subjects who are suspected of lowered, abnormal, CSF A $\beta$ levels ("A $\beta$ positive subjects") consistent with the presence of AD pathology. The algorithm includes sex, 7 cognitive tests measured with the NeuroCart (MMT, VVLT, finger tapping, NBack, SART, Face, and EEG), and one plasma biomarker (YKL-40) and was successful in predicting CSF A $\beta+$ in healthy elderly with a sensitivity of $70.82 \%$ and specificity of $89.25 \%$. When using this algorithm, $70 \%$ fewer lumbar punctures will have to be performed to enroll subjects based on lowered Abeta CSF. The overall subject burden and costs of trials will reduce as fewer lumbar punctures will need to be performed. This may also increase subject's willingness to participate.

Four classification algorithms (random forest, logistic regression, support vector machine classifier, and a $\mathrm{K}$ nearest neighbors classifier) were used to classify $A \beta$ positivity. A comparison of classification models is necessary to identify a model that best fits the data. Logistic regression outperformed the other algorithms in terms of accuracy, precision, and recall. The logistic regression model is ideal for $A \beta$ positivity classification as it provides an estimation of the association between the predictor and the outcome. Palmqvist et al. [47] and Jang et al. [48] have also demonstrated the use of logistic regression to reliably dichotomize amyloid status using plasma. This further supports the notion that logistic regression can use multimodal non-invasive cognitive and blood-based biomarkers to stratified enrollment of subjects with preclinical AD into clinical trials. In this study, 200 healthy elderly were included of which 154 were eventually included in the model. This is a satisfactory amount of subjects to support the conclusion of this study. For the logistic regression classifier, we have selected 0.5 to be the probabilistic threshold for classifying a patient as $\mathrm{A} \beta+$ or $\mathrm{A} \beta-$. Using the ROC curve (Fig. 1), a researcher may choose a different threshold depending on what they choose to prioritize, the true positive rate (sensitivity) or the false positive rate (1-specificity)).

Approximately 50 subjects is an acceptable number for a proof-Of-concept study of a novel compound; 20-80 subjects is common in phase one trials according to the FDA [49]. Based on the $27.3 \% \mathrm{~A} \beta$ positivity in our study, we estimate that in a new group of 220 healthy elderly, 71 subjects will be $A \beta+$. The algorithm will identify 66 subjects as having $A \beta+C S F$. Due to the sensitivity of $70.82 \%, 21 \mathrm{~A} \beta$ + subjects would not be identified as such. Also, $16 \mathrm{~A} \beta$-subjects would wrongfully be identified as $A \beta+$ which results in 50 truly $A \beta+$ subjects. Using the algorithm would reduce the number of lumbar punctures in healthy elderly by $70 \%$, i.e., 66 lumbar 


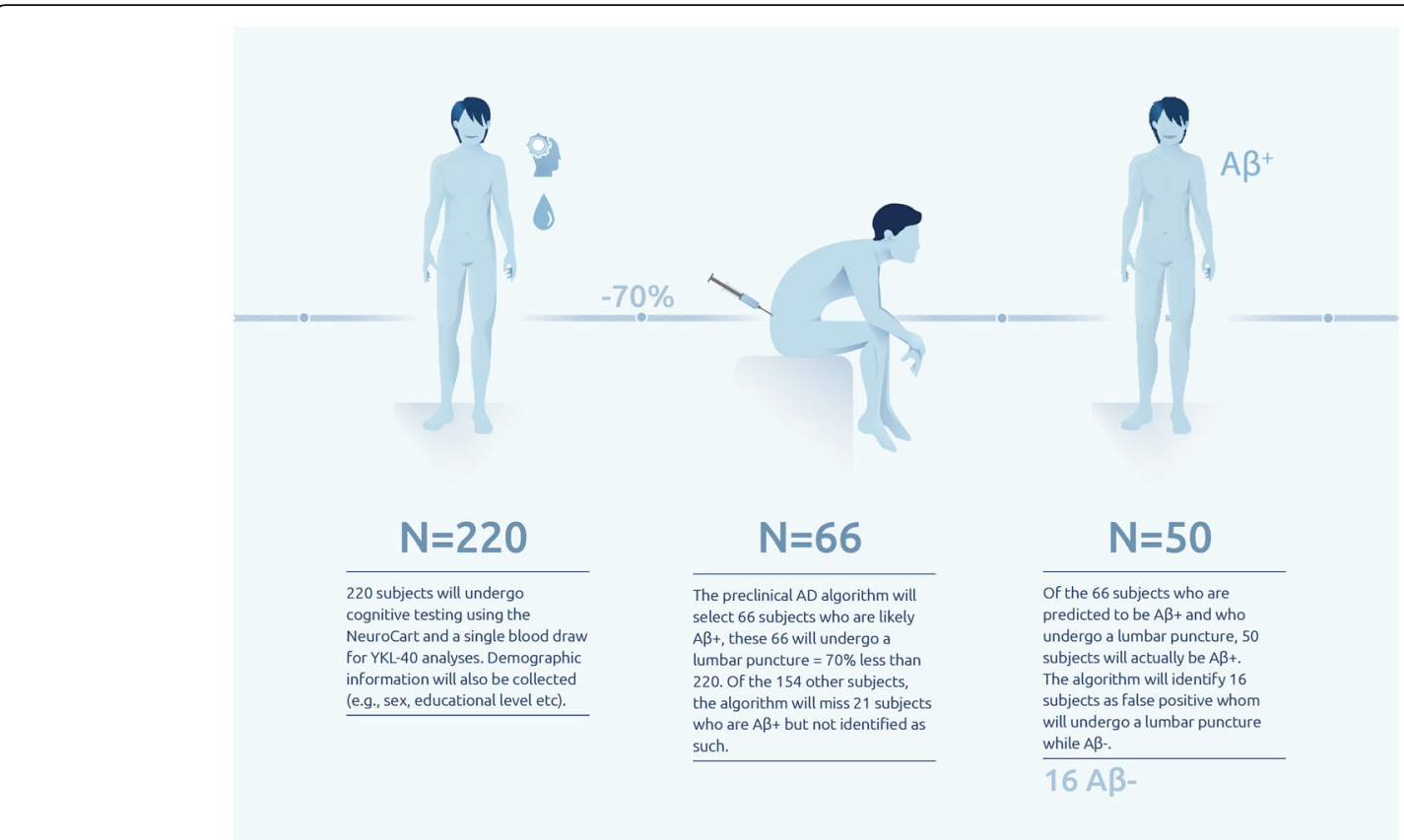

Fig. 2 Visualization of reduction of lumbar punctures using the algorithm

punctures instead of 220. As this algorithm is designed to select healthy elderly with A $\beta$ CSF concentrations consistent with $\mathrm{AD}$, having a $100 \%$ accuracy is of no importance, contrary to when using a test or an algorithm for diagnostic purposes. We would not perform unnecessary lumbar punctures in $89.25 \%$ patients with an increased chance of being $A \beta-$. In our opinion, this decrease in overall burden justifies the use of such an algorithm for subject selection for trial.

Other studies developed algorithms focused on predicting the progression to dementia due to $\operatorname{AD}[50,51]$, the classification of different stages of $\mathrm{AD}[52,53]$, and for the diagnosis of AD in the early stages [54]. These algorithms were developed for diagnostic purpose rather than for clinical trial participation, such as the one described in our study. Also, the data used in these algorithms were collected in clinical settings such as behavioral observation, clinical presentation, and MRI data. When selecting healthy elderly for clinical trial participation, this information is commonly not available. Others have tried to identify healthy subjects with amyloid pathology using considerably burdensome and costly MRI data [55, 56]. Khan et al. (2018) suggests an algorithm for preclinical diagnosis of AD based on a combination of three $\mathrm{AD}$ biomarkers: neuroimaging, genetic markers, and abnormalities in CSF A $1-42$, $t$-tau, and $p-$ tau (the gold standard for the diagnosis of AD). However, as mentioned before, data from neuroimaging is not commonly available and far more costly and time consuming than the tests used in our algorithm.
Reduction of the number of lumbar punctures performed in healthy subjects is of great value to increase participation willingness in healthy elderly and to lower overall subject burden. A comparable study to this current study showed that $A \beta$ positivity (confirmed by either CSF or PET-MRI) can be predicted by a combination of demographic variables, APOE status, baseline cognition, and 24-month follow-up rates [57]. A 24month follow-up is usually not available and gathering follow up information on healthy subjects before the start of a clinical trial is too time consuming.

Accumulation of $A \beta$ plaques in the brain associated with lowered levels of $A \beta$ in CSF is still seen as the main pathological cause of AD. Various clinical trials have therefore focused on reducing $A \beta$ plaques in the brain. Where reducing $A \beta$ has been successful, lowering the prevalence of dementia due to $\mathrm{AD}$ has not been a result. Huang et al. (2020) reported 9 failed phase 3 antiamyloid trails since 2016 with 6 different compounds [58]. Two of these trails were performed in subjects with preclinical AD, both with BACE inhibitors $[17,18]$ and both were discontinued due to either toxicity or lack of efficacy. Researchers claim that interfering early in the disease process will probably result in higher efficacy than when the clinical disease process has already started, evidenced by a diagnosis of preclinical $\mathrm{AD}$ or MCI. Looking at the inclusion criteria of the aforementioned studies shows that healthy elderly with CSF A $\beta$ levels consistent with $\mathrm{AD}$ have been selected for participation. Healthy elderly are defined as having a clinical 
interview, namely the clinical dementia rating scale (CDR) of 0. Using the CDR total score is well accepted in clinical research and is widely used for clinical diagnosis of AD [59]. Still, very subtle cognitive changes are not detected using this crude screening tool. Using the algorithm proposed in this article will help to better select trial participants by including diverse cognitive assessments instead of the more general cognitive score of the CDR.

Shifting focus from invasive measurements (CSF, PET-MRI) to blood-based biomarkers for $\mathrm{AD}$ has been a major topic in research as new technics have been developed claiming to be ultrasensitive to detecting AD-related proteins [24]. Using a blood test would make it more accessible to diagnose patients but also to identify possible trial participants. Challenges in the use of blood-based AD biomarkers are the different biological system compared to the CSF system, use of different analytical methods (ELISA, Simoa, etc.), and the specificity for AD of these biomarkers [27]. Specifying pre-AD stages with the use of blood-based biomarkers has yet to be standardized. The preclinical AD algorithm created in this study includes only one blood-based biomarker (YKL-40) and the limitations of using blood-based biomarkers are therefore minor. The use of a different analytical method may alter the outcome of the analysis slightly and therefore could have led to a different composition of the algorithm. This should be kept in mind when comparing the outcome of this study to those of other studies. The combination of blood-based biomarkers with genetic information and cognitive assessments appears to be a powerful tool in preselection of preclinical $\mathrm{AD}$ subjects in clinical trials.

Four out of the seven NeuroCart tasks that are included in the algorithm are memory tasks. Loss of memory early on in the disease process is common for (amnestic) MCI and often lead to the AD diagnosis [60]. Especially the visual verbal learning task is important for the algorithm to differentiate between preclinical $A D$ and healthy elderly. Visual and verbal memory problems are common in $\mathrm{AD}$ [61] and have also been reported in preclinical AD [62, 63].

Reducing the number of lumbar punctures in healthy subjects and the additional benefits for clinical research must be weighed against the ethical consequences of identifying healthy subjects with an elevated risk of developing $\mathrm{AD}$, which at this moment is an untreatable disease. Approximately $53 \%$ of subjects fulfilling the criteria of preclinical AD will actually develop MCI or AD [64]. When selecting trial subjects based on specific biomarkers, these subjects will become aware that they have CSF A $\beta$ levels consistent with AD. The development of
$\mathrm{A} \beta$ plaques in the brain and eventually developing $\mathrm{AD}$ can be a 20- to 30-year-long process [19]. This is a substantial amount of time to be concerned about a disease that one might develop. Knowledge about predispositions to develop a disease can even have financial consequences and reduce health benefits as people might not be hired for certain jobs and health insurances may increase insurance premium. Nevertheless, studying cognitively healthy elderly is important as treatment in a predisease phase might prevent or retard the process of developing clinically overt Alzheimer's dementia. With the ultimate goal of preventing $\mathrm{AD}$, the need to include preclinical subjects in clinical studies is vital.

\section{Limitations}

Among the limitations is that a logistic algorithm was used which cannot incorporate incomplete datasets [65]. Hence, the model will fail to predict a class if a subject is missing a single feature. Missing data is not uncommon in research, especially when cognitive tests are performed. Benefits of using this model however proceed this limitation. Inconclusiveness about the validity of blood based biomarkers can also be regarded as a limitation of this study. This study only includes one plasma biomarker which reduces the inconvenience. The ethical consequences of using an algorithm like ours in healthy elderly should always be taken into account and could be regarded as a limitation. The study population is a relatively highly educated group. This might not be completely representative with regards to the cognitive performance of an average population.

\section{Conclusion}

This algorithm would allow for a 70\% reduction of lumbar punctures needed to identify subjects with abnormal CSF A $\beta$ levels consistent with AD. We have identified an algorithm that is able to preselect healthy elderly who are more likely to have $A \beta$ CSF levels consistent with AD. Using this algorithm, fewer lumbar punctures will have to be performed when selecting subjects for clinical trials. The use of this algorithm can be expected to lower overall subject burden and costs of identifying subjects with preclinical $\mathrm{AD}$ and therefore of total study costs.

\footnotetext{
Abbreviations

A $\beta$ : Amyloid beta; $A \beta+$ : Amyloid beta positive; AD: Alzheimer's disease; Apoe: Apolipoprotein; AUC: Area under the curve; CHDR: Centre for Human Drug Research; CSF: Cerebrospinal fluid; CDR: Clinical dementia rating scale; EEG: Electro encephalography; IADL: Instrumental activities of daily living scale; MCl: Mild cognitive impairment; MMSE: Mini mental state examination; MMT: Milner Maze test; MRI: Magnetic reasoning image; NfL: Neurofilament light; SART: Sustained attention to response task; PET: Positron emission tomography; ROC: Receiver operating characteristics; WLT: Visual verbal learning test; VIF: Variance inflation factor
} 


\section{Supplementary Information}

The online version contains supplementary material available at https://doi. org/10.1186/s13195-021-00874-9.

Additional file 1. STARD flow chart.

\section{Acknowledgements}

The authors thank Christel Havers, Kimberley Versteegen, Jacky Zuiddam, and Renate van Rijt for their contributions to the clinical execution of the study. We also like to thank Folkert van Meurs for designing Fig. 2.

\section{Authors' contributions}

The study design, collection of data, and analysis and interpretation of the data has been a mutual effort of the authors. The authors read and approved the final manuscript.

\section{Funding}

This study was investigator initiated and funded by CHDR.

\section{Availability of data and materials}

The datasets generated during this study are not available by request.

\section{Declarations}

\section{Ethics approval and consent to participate}

The study was approved by the ethics committee of the Leiden University Hospital, the Netherlands. The study was conducted according to the Dutch act on Medical Research Involving Human Subjects (WMO) and in compliance with Good Clinical Practice (ICH-GCP) and the Declaration of Helsinki. The trial was retrospectively registered in the international trial register ISRCTN79036545. Written and informed consent was obtained from all the included participants.

\section{Consent for publication}

All authors consent to publication of the study.

\section{Competing interests}

The authors declare that they have no competing interests.

Received: 11 March 2021 Accepted: 4 July 2021

Published online: 17 July 2021

\section{References}

1. Amieva H, le Goff M, Millet X, Orgogozo JM, Pérès K, Barberger-Gateau P, et al. Prodromal Alzheimer's disease: successive emergence of the clinical symptoms. Ann Neurol. 2008;64(5):492-8. https://doi.org/10.1002/ana.21509.

2. Dubois B, Feldman HH, Jacova C, DeKosky ST, Barberger-Gateau P, Cummings J, et al. Research criteria for the diagnosis of Alzheimer's disease: revising the NINCDS-ADRDA criteria. Lancet Neurol. 2007;6(8):734-46. https://doi.org/10.1016/S1474-4422(07)70178-3.

3. Price JL, McKeel DW Jr, Buckles VD, Roe CM, Xiong C, Grundman M, et al. Neuropathology of nondemented aging: presumptive evidence for preclinical Alzheimer disease. Neurobiol Aging. 2009;30(7):1026-36. https:// doi.org/10.1016/j.neurobiolaging.2009.04.002.

4. Sevigny J, Chiao P, Bussière T, Weinreb PH, Williams L, Maier M, et al. The antibody aducanumab reduces Abeta plaques in Alzheimer's disease. Nature. 2016;537(7618):50-6. https://doi.org/10.1038/nature19323.

5. Schneider L. A resurrection of aducanumab for Alzheimer's disease. Lancet Neurol. 2020;19(2):111-2. https://doi.org/10.1016/S1474-4422(19)30480-6.

6. FDA. FDA grants accelerated approval for Alzheimer's drug. 2021 content current as of 07Jun2018 [cited 2021 18June].

7. Lemere CA, Masliah E. Can Alzheimer disease be prevented by amyloid-beta immunotherapy? Nat Rev Neurol. 2010;6(2):108-19. https://doi.org/10.1038/ nrneurol.2009.219.

8. Rinne JO, Brooks DJ, Rossor MN, Fox NC, Bullock R, Klunk WE, et al. 11C-PiB PET assessment of change in fibrillar amyloid-beta load in patients with Alzheimer's disease treated with bapineuzumab: a phase 2, double-blind, placebo-controlled, ascending-dose study. Lancet Neurol. 2010;9(4):363-72. https://doi.org/10.1016/S1474-4422(10)70043-0.
9. Leyhe T, Andreasen N, Simeoni M, Reich A, von Arnim CAF, Tong X, et al. Modulation of beta-amyloid by a single dose of GSK933776 in patients with mild Alzheimer's disease: a phase I study. Alzheimers Res Ther. 2014;6(2):19. https://doi.org/10.1186/alzrt249.

10. Salloway S, Sperling R, Fox NC, Blennow K, Klunk W, Raskind M, et al. Two phase 3 trials of bapineuzumab in mild-to-moderate Alzheimer's disease. N Engl J Med. 2014;370(4):322-33. https://doi.org/10.1056/NEJMoa1304839.

11. Selkoe DJ. Alzheimer disease and aducanumab: adjusting our approach. Nat Rev Neurol. 2019;15(7):365-6. https://doi.org/10.1038/s41582-019-0205-1.

12. Swanson CJ, Zhang Y, Dhadda S, Wang J, Kaplow J, Lai RYK, et al. A randomized, double-blind, phase $2 \mathrm{~b}$ proof-of-concept clinical trial in early Alzheimer's disease with lecanemab, an anti-A $\beta$ protofibril antibody. Alzheimers Res Ther. 2021;13(1):80. https://doi.org/10.1186/s13195-02100813-8.

13. Chiu MJ, Yang SY, Horng HE, Yang CC, Chen TF, Chieh JJ, et al. Combined plasma biomarkers for diagnosing mild cognition impairment and Alzheimer's disease. ACS Chem Neurosci. 2013;4(12):1530-6. https://doi. org/10.1021/cn400129p.

14. Jack CR Jr, Knopman DS, Jagust WJ, Petersen RC, Weiner MW, Aisen PS, et al. Tracking pathophysiological processes in Alzheimer's disease: an updated hypothetical model of dynamic biomarkers. Lancet Neurol. 2013; 12(2):207-16. https://doi.org/10.1016/S1474-4422(12)70291-0.

15. Jack CR Jr, Knopman DS, Jagust WJ, Shaw LM, Aisen PS, Weiner MW, et al. Hypothetical model of dynamic biomarkers of the Alzheimer's pathological cascade. Lancet Neurol. 2010;9(1):119-28. https://doi.org/10.1016/S14 74-4422(09)70299-6.

16. Jack CR Jr, Bennett DA, Blennow K, Carrillo MC, Dunn B, Haeberlein SB, et al. NIA-AA Research Framework: toward a biological definition of Alzheimer's disease. Alzheimers Dement. 2018;14(4):535-62. https://doi.org/10.1016/j.ja lz.2018.02.018

17. Timmers M, Streffer JR, Russu A, Tominaga Y, Shimizu H, Shiraishi A, et al. Pharmacodynamics of atabecestat (JNJ-54861911), an oral BACE1 inhibitor in patients with early Alzheimer's disease: randomized, double-blind, placebo-controlled study. Alzheimers Res Ther. 2018;10(1):85. https://doi. org/10.1186/s13195-018-0415-6.

18. Neumann U, et al. EMBO Mol Med. 2018;10(11).

19. Jansen WJ, Ossenkoppele R, Knol DL, Tijms BM, Scheltens P, Verhey FR, et al. Prevalence of cerebral amyloid pathology in persons without dementia a meta-analysis. JAMA. 2015;313(19):1924-38. https://doi.org/10.1001/jama.201 5.4668 .

20. Zwan M, van Harten A, Ossenkoppele R, Bouwman F, Teunissen C, Adriaanse $\mathrm{S}$, et al. Concordance between cerebrospinal fluid biomarkers and [C-11] PIB PET in a memory clinic cohort. J Alzheimers Dis. 2014;41(3):801-7. https://doi.org/10.3233/JAD-132561.

21. Zetterberg H, Burnham SC. Blood-based molecular biomarkers for Alzheimer's disease. Molecular Brain. 2019;12(1):26. https://doi.org/10.1186/ s13041-019-0448-1.

22. Olsson B, Lautner R, Andreasson U, Öhrfelt A, Portelius E, Bjerke M, et al. CSF and blood biomarkers for the diagnosis of Alzheimer's disease: a systematic review and meta-analysis. Lancet Neurol. 2016;15(7):673-84. https://doi. org/10.1016/S1474-4422(16)00070-3.

23. Verberk IMW, Slot RE, Verfaillie SCJ, Heijst H, Prins ND, van Berckel BNM, et al. Plasma amyloid as prescreener for the earliest Alzheimer pathological changes. Ann Neurol. 2018;84(5):648-58. https://doi.org/10.1002/ana.25334.

24. Janelidze S, Stomrud E, Palmqvist S, Zetterberg $H$, van Westen D, Jeromin A, et al. Plasma beta-amyloid in Alzheimer's disease and vascular disease. Sci Rep. 2016;6(1):26801. https://doi.org/10.1038/srep26801.

25. Mielke MM, Hagen CE, Xu J, Chai X, Vemuri P, Lowe VJ, et al. Plasma phospho-tau181 increases with Alzheimer's disease clinical severity and is associated with tau- and amyloid-positron emission tomography. Alzheimers Dement. 2018;14(8):989-97. https://doi.org/10.1016/j.jalz.2018. 02.013.

26. Lewczuk P, Ermann N, Andreasson U, Schultheis C, Podhorna J, Spitzer P, et al. Plasma neurofilament light as a potential biomarker of neurodegeneration in Alzheimer's disease. Alzheimers Res Ther. 2018;10(1): 71. https://doi.org/10.1186/s13195-018-0404-9.

27. Hampel H, O'Bryant SE, Molinuevo JL, Zetterberg H, Masters CL, Lista S, et al. Blood-based biomarkers for Alzheimer disease: mapping the road to the clinic. Nat Rev Neurol. 2018;14(11):639-52. https://doi.org/10.1038/s41582-01 8-0079-7. 
28. Lewczuk P, Mroczko B, Fagan A, Kornhuber J. Biomarkers of Alzheimer's disease and mild cognitive impairment: a current perspective. Adv Med Sci. 2015;60(1):76-82. https://doi.org/10.1016/j.advms.2014.11.002.

29. Rissin DM, Kan CW, Campbell TG, Howes SC, Fournier DR, Song L, et al. Single-molecule enzyme-linked immunosorbent assay detects serum proteins at subfemtomolar concentrations. Nat Biotechnol. 2010;28(6):595-9. https://doi.org/10.1038/nbt.1641.

30. Muszynski $P$, et al. YKL-40 as a potential biomarker and a possible target in therapeutic strategies of Alzheimer's disease. Curr Neuropharmacol. 2017.

31. Antonell A, Mansilla A, Rami L, Lladó A, Iranzo A, Olives J, et al. Cerebrospinal fluid level of YKL-40 protein in preclinical and prodromal Alzheimer's disease. J Alzheimers Dis. 2014;42(3):901-8. https://doi.org/1 0.3233/JAD-140624.

32. Kester MI, Teunissen CE, Sutphen C, Herries EM, Ladenson JH, Xiong C, et al. Cerebrospinal fluid VILIP-1 and YKL-40, candidate biomarkers to diagnose, predict and monitor Alzheimer's disease in a memory clinic cohort. Alzheimers Res Ther. 2015;7(1):59. https://doi.org/10.1186/s13195-0150142-1.

33. Groeneveld GJ, Hay JL, Van Gerven JM. Measuring blood-brain barrier penetration using the NeuroCart, a CNS test battery. Drug Discov Today Technol. 2016;20:27-34. https://doi.org/10.1016/j.ddtec.2016.07.004.

34. Borland RG, Nicholson AN. Visual motor co-ordination and dynamic visual acuity. Br J Clin Pharmacol. 1984;18(Suppl 1):69S-72S. https://doi.org/1 0.1111/j.1365-2125.1984.tb02583.x

35. Lezak MD, Howieson DB, Loring DW. Neuropyschological assessment, vol. 4th. New York: Oxford University Press; 2004

36. de Haas SL, Franson KL, Schmitt JAJ, Cohen AF, Fau JB, Dubruc C, et al. The pharmacokinetic and pharmacodynamic effects of SL65.1498, a GABA-A alpha2,3 selective agonist, in comparison with lorazepam in healthy volunteers. J Psychopharmacol. 2009;23(6):625-32. https://doi.org/10.1177/ 0269881108092595.

37. Milner B. Visually-guided maze-learning in man - effects of bilateral hippocampal, bilateral frontal, and unilateral cerebral-lesions. Neuropsychologia. 1965;3(4):317-38. https://doi.org/10.1016/0028-3932 (65) $90005-9$

38. Rombouts SARB, et al. Alterations in brain activation during cholinergic enhancement with rivastigmine in Alzheimer's disease. J Neurol Neurochir Psychiatr. 2002;73(6):665-71. https://doi.org/10.1136/jnnp.73.6.665.

39. Dudschig C, Jentzsch I. Speeding before and slowing after errors: is it all just strategy? Brain Res. 2009;1296:56-62. https://doi.org/10.1016/j.brainres.2009. 08.009.

40. Andrew JM. Delinquents and the tapping test. J Clin Psychol. 1977;33(3): 786-91. https://doi.org/10.1002/1097-4679(197707)33:3<786::AID-JCLP22 70330340>3.0.CO;2-X.

41. Molitor RJ, Ko PC, Ally BA. Eye movements in Alzheimer's disease. J Alzheimers Dis. 2015;44(1):1-12. https://doi.org/10.3233/JAD-141173.

42. Rossini PM, Rossi S, Babiloni C, Polich J. Clinical neurophysiology of aging brain: from normal aging to neurodegeneration. Prog Neurobiol. 2007;83(6): 375-400. https://doi.org/10.1016/j.pneurobio.2007.07.010.

43. Morris JC. The Clinical Dementia Rating (CDR): current version and scoring rules. Neurology. 1993;43(11):2412-4. https://doi.org/10.1212/ wnl.43.11.2412-a.

44. Lawton MP, Brody EM. Assessment of older people: self-maintaining and instrumental activities of daily living. Gerontologist. 1969;9(3):179-86. https://doi.org/10.1093/geront/9.3 Part_1.179.

45. Cui Y, Sachdev PS, Lipnicki DM, Jin JS, Luo S, Zhu W, et al. Predicting the development of mild cognitive impairment: a new use of pattern recognition. Neuroimage. 2012;60(2):894-901. https://doi.org/10.1016/j. neuroimage.2012.01.084

46. Zhang D, Wang Y, Zhou L, Yuan H, Shen D, Alzheimer's Disease Neuroimaging Initiative. Multimodal classification of Alzheimer's disease and mild cognitive impairment. Neuroimage. 2011;55(3):856-67. https://doi.org/1 0.1016/j.neuroimage.2011.01.008.

47. Palmqvist $\mathrm{S}$, et al. Performance of fully automated plasma assays as screening tests for Alzheimer disease-related beta-amyloid status. JAMA Neurol. 2019.

48. Jang H, Park J, Woo S, Kim S, Kim HJ, Na DL, et al. Prediction of fast decline in amyloid positive mild cognitive impairment patients using multimodal biomarkers. Neuroimage Clin. 2019;24:101941. https://doi.org/10.1016/j.nicl.2 019.101941.

49. FDA. 2021 content current as of 04Jan2018 [cited 2021 14Jan].
50. Lewczuk P, et al. Validation of the Erlangen score algorithm for the prediction of the development of dementia due to Alzheimer's disease in pre-dementia subjects. J Alzheimers Dis. 2015;48(2):433-41. https://doi.org/1 $0.3233 / J A D-150342$

51. Lutz MW, et al. A genetics-based biomarker risk algorithm for predicting risk of Alzheimer's disease. Alzheimers Dement. 2016:2(1):30-44.

52. Ahmad F, Dar WM. Classification of Alzheimer's disease stages: an approach using PCA-based algorithm. Am J Alzheimers Dis Dement. 2018;33(7):433-9. https://doi.org/10.1177/1533317518790038.

53. An N, Ding H, Yang J, Au R, Ang TFA. Deep ensemble learning for Alzheimer's disease classification. J Biomed Inform. 2020;105:103411. https:// doi.org/10.1016/j.jbi.2020.103411.

54. Zheng $W$, et al. Application of generalized split linearized bregman iteration algorithm for Alzheimer's disease prediction. Aging. 2020;12(7):6206-24. https://doi.org/10.18632/aging.103017.

55. Mielke MM, Wiste HJ, Weigand SD, Knopman DS, Lowe VJ, Roberts RO, et al. Indicators of amyloid burden in a population-based study of cognitively normal elderly. Neurology. 2012;79(15):1570-7. https://doi.org/10.1212/WNL. Ob013e31826e2696.

56. Casamitjana A, et al. MRI-based screening of preclinical Alzheimer's disease for prevention clinical trials. J Alzheimers Dis. 2018;64(4):1099-112. https:// doi.org/10.3233/JAD-180299.

57. Insel PS, Palmqvist S, Mackin RS, Nosheny RL, Hansson O, Weiner MW, et al. Assessing risk for preclinical beta-amyloid pathology with APOE, cognitive, and demographic information. Alzheimers Dement. 2016;4:76-84. https:// doi.org/10.1016/j.dadm.2016.07.002

58. Huang LK, Chao SP, Hu CJ. Clinical trials of new drugs for Alzheimer disease. J Biomed Sci. 2020;27(1).

59. Huang HC, et al. Diagnostic accuracy of the Clinical Dementia Rating Scale for detecting mild cognitive impairment and dementia: a bivariate metaanalysis. Int J Geriatr Psychiatry. 2020.

60. Jahn H. Memory loss in Alzheimer's disease. Dialogues Clin Neurosci. 2013; 15(4):445-54.

61. Tromp D, et al. Episodic memory in normal aging and Alzheimer disease: insights from imaging and behavioral studies. Ageing Res Rev. 2015;24(Pt B): 232-62.

62. Baker JE, Lim YY, Jaeger J, Ames D, Lautenschlager NT, Robertson J, et al. Episodic memory and learning dysfunction over an 18-month period in preclinical and prodromal Alzheimer's disease. J Alzheimers Dis. 2018;65(3): 977-88. https://doi.org/10.3233/JAD-180344

63. Stricker NH, Lundt ES, Albertson SM, Machulda MM, Pudumjee SB, Kremers WK, et al. Diagnostic and prognostic accuracy of the Cogstate Brief Battery and Auditory Verbal Learning Test in preclinical Alzheimer's disease and incident mild cognitive impairment: implications for defining subtle objective cognitive impairment. J Alzheimers Dis. 2020;76(1):261-74. https:// doi.org/10.3233/JAD-200087.

64. Parnetti L, Chipi E, Salvadori N, D'Andrea K, Eusebi P. Prevalence and risk of progression of preclinical Alzheimer's disease stages: a systematic review and meta-analysis. Alzheimers Res Ther. 2019;11(1). https://doi.org/10.1186/ s13195-018-0459-7.

65. Jiang $W$, Josse J, Lavielle M. Logistic regression with missing covariates parameter estimation, model selection and prediction within a jointmodeling framework. Comput Stat Data Anal. 2020;145:106907. https://doi. org/10.1016/j.csda.2019.106907

\section{Publisher's Note}

Springer Nature remains neutral with regard to jurisdictional claims in published maps and institutional affiliations. 